\title{
Térmitas (Blattodea) em plantio homogêneo de Castanha-do-Pará (Bertholletia excelsa Bonpl.) no sul da Amazônia
}

\author{
Termites (Blattodea) in a homogen plantation of Pará Nut (Bertholletia excelsa \\ Bonpl.) in the south Amazon
}

\author{
Marcus Henrique Martins e Silva ${ }^{\mathrm{I}}$, Juliana Garlet ${ }^{\mathrm{II}}$, \\ Fernando Luiz Silva ${ }^{\mathrm{III}}$, Carla da Silva Paula ${ }^{\mathrm{IV}}$
}

\begin{abstract}
Resumo
Plantios homogêneos de Castanha-do-Pará na região Norte de Mato Grosso apresentam elevado potencial, devido às características edafoclimáticas locais e à importância econômica dessa espécie florestal. Nesse aspecto, estudar a ocorrência de térmitas e sua distribuição espacial em plantios homogêneos de Castanha-do-Pará torna-se de extrema relevância, tendo em vista a carência de estudos entomológicos para esses cultivos, bem como para subsidiar informações técnicas acerca da dinâmica desses insetos nesses ambientes. Assim, o objetivo do presente trabalho foi avaliar a ocorrência e a distribuição espacial de térmitas subterrâneos em um plantio homogêneo de Castanha-do-Pará no Norte de Mato Grosso. O estudo foi desenvolvido em um plantio homogêneo de 28 ha, com aproximadamente 16 anos de idade, onde se estabeleceu amostragem em gride com 40 pontos amostrais. Em cada ponto amostral foram instaladas iscas celulósicas inseridas no solo a $20 \mathrm{~cm}$ de profundidade, as quais permaneceram em campo por 40 dias. Foram identificados dois gêneros de térmitas, Heterotermes e Nasutitermes, e um total de 1.405 indivíduos, sendo $396(28,1 \%)$ pertencentes ao gênero Nasutitermes, e $1.009(71,8 \%)$ à espécie Heterotermes tenuis. O padrão de distribuição espacial dos térmitas no castanhal é do tipo agregado. A ocorrência da espécie Heterotermes tenuis no plantio homogêneo de Castanha-do-Pará deve ser avaliada com atenção, visto que essa espécie pode se tornar potencial praga na região.
\end{abstract}

Palavras-chave: Cupins; Heterotermes; Silvicultura; Amazônia

\begin{abstract}
Homogeneous plantations of Castanha-do-Pará in the northern region of Mato Grosso have high potential due to the local edaphoclimatic characteristics and the economic importance of this forest species. In this aspect, studying the occurrence of termites and their spatial distribution in homogeneous plantations of Pará Nut has become extremely relevant considering the lack of entomological studies for these crops and the need to subsidize technical information about the dynamics of these insects in these environments. Thus, the objective of the present study was to evaluate the occurrence and spatial distribution of underground termites in a homogeneous plantation of Pará Nut in northern Mato Grosso. The study was carried out in a 16-year-old homogeneous plantation covering an area of $28 \mathrm{ha}$; sampling was performed in grids with 40 sampling points. At each sampling point, cellulosic baits were inserted into the soil at a depth of $20 \mathrm{~cm}$ and remained in the field for 40 days. Two termite genera were identified, Heterotermes and Nasutitermes, with a total of 1,405 individuals; of these, 396 (28.1\%) belonged to the genus Nasutitermes and $1,009(71.8 \%)$ to the species Heterotermes tenuis. The spatial distribution pattern of termites in Pará Nut is of the aggregate type. The occurrence of the species Heterotermes tenuis in homogeneous plantings of Pará Nut must be carefully evaluated, as this species is a potential pest in this region.

Keywords: Termites; Heterotermes; Silviculture; Amazon

\footnotetext{
Engenheiro Agrônomo, Me., Instituto Federal de Mato Grosso - Campus Alta Floresta, Rod. MT-208, s/n - lote 143-A, CEP 78580-000, Alta Floresta (MT), Brasil. marcus.silva@alf.ifmt.edu.br (ORCID: 0000-0002-9446-1064) CEP 78580-000, Alta Floresta (MT), Brasil. julianagarlet@unemat.br (ORCID: 0000-0002-0791-7060) Brasil. fernando.silva@alf.ifmt.edu.br (ORCID: 0000-0002-7897-0395) gmail.com (ORCID: 0000-0002-6328-5395)
}

II Engenheira Florestal, Dra., Professora da Universidade do Estado de Mato Grosso - Campus Alta Floresta. Rod. MT-208, Km 147, Jardim Tropical,

III Zootecnista, Me., Instituto Federal de Mato Grosso - Campus Alta Floresta, Rod. MT-208, s/n - lote 143-A, CEP 78580-000, Alta Floresta (MT),

IV Engenheira Sanitarista e Ambiental, Ma., Pesquisadora Autônoma, Rua F-3, Setor F, nº 303, CEP 78580-000, Alta Floresta (MT), Brasil. carlapaula22@
\end{abstract}




\section{Introdução}

A implantação de sistemas homogêneos de Castanha-do-Pará (Bertholletia excelsa Bonpl.) apresenta um grande potencial na região Norte de Mato Grosso, dadas as condições edafoclimáticas favoráveis ao cultivo dessa espécie, a demanda de mercado e a valorização das amêndoas. O estado respondeu, na safra 2018, por 6,3\% da produção nacional (2.179 toneladas), em razão, sobretudo, dos castanhais que compõem a região norte mato-grossense (IBGE, 2019). Os desafios para Mato Grosso são o investimento em pesquisas, novas tecnologias produtivas e de manejo, o fortalecimento e a consolidação da infraestrutura da cadeia produtiva, a potencialização de iniciativas de reflorestamento e plantio de mudas precoces e a ampliação de um programa de exploração sustentável de Castanhado-Pará (FERNANDES, 2018).

O cultivo da Castanha-do-Pará se torna relevante também pelo fato de que o desflorestamento tem comprometido a sustentabilidade dos castanhais nativos para a exploração do extrativismo das amêndoas. Homma et al. (2014) apontam um crescimento dos castanhais plantados, com maior tecnificação, estratégias de manejo e uso de práticas agrícolas sustentáveis.

Em castanheiras nativas estudadas na região oeste do Pará, Scoles et al. (2016) verificaram danos severos por térmitas (cupins). No entanto, em sistemas homogêneos de Castanha-do-Pará, informações acerca da incidência e de danos por térmitas são escassas. A avaliação da ocorrência e da distribuição espacial nesses agroecossistemas possibilita o avanço na sistematização de técnicas de manejo integrado de pragas nessa cultura. Além disso, como bem destacam Sales et al. (2010), a falta de pesquisas sobre térmitas, sobretudo em relação às suas interações em florestas plantadas, é um entrave ao reconhecimento da importância desses organismos como insetos indispensáveis aos processos ecológicos.

Os térmitas (Blattodea: Termitoidea) são agentes detritívoros que possuem grande importância econômica em sistemas florestais. No Brasil, já foi registrada a ocorrência de 300 espécies que são pertencentes às famílias Kalotermitidae, Rhinotermitidae, Serritermitidae e Termitidae. Em determinadas condições ecológicas e de manejo, esses insetos podem se tornar pragas de madeira e de outros materiais celulósicos. Representam um dos grupos mais dominantes da fauna de ecossistemas tropicais e exercem importante papel na ciclagem de nutrientes e na formação do solo (CONSTANTINO, 2015).

No setor florestal, os térmitas subterrâneos são de grande importância por causarem danos ao sistema radicular de árvores de valor comercial (PERES FILHO et al., 2010). Esse grupo de térmitas existe em todas as regiões tropicais e subtropicais, fazem túneis pelo solo e constroem ninhos e galerias com solo e material fecal, sendo todas as espécies xilófagas (CONSTANTINO, 2015). Além disso, deve-se considerar que a compreensão da dinâmica de ocorrência desses agentes nos sistemas florestais é importante também para o desenvolvimento de estratégias de manejo integrado e de sustentabilidade da produção.

Dessa forma, o objetivo do presente trabalho é avaliar a ocorrência e a distribuição espacial de térmitas em um plantio homogêneo de Castanha-do-Pará no sul da Amazônia.

\section{Material e métodos}

\section{Área de Estudo}

A pesquisa foi desenvolvida em um plantio homogêneo de Castanha-do-Pará de aproximadamente 16 anos de idade (coordenadas: 9 ${ }^{\circ} 40^{\prime} 19.33^{\prime \prime S}$ e $56^{\circ} 27^{\prime} 32.58^{\prime \prime} \mathrm{W}$ ), localizado no município de Paranaíta, Mato Grosso. A área total do castanhal é de 28 ha, densidade de plantio de 278 plantas/ha, espaçamento de $6 \mathrm{~m} \times 6 \mathrm{~m}$, com indivíduos de DAP médio de $25 \mathrm{~cm}$, e altura média de $8 \mathrm{~m}$, tendo no entorno de todo o plantio áreas de pastagens. O solo da área de estudo é classificado como Latossolo Vermelho Distrófico e clima Aw com estações bem definidas, ocorrendo chuvas de verão e inverno seco (ALVARES et al., 2014). 


\section{Procedimento Amostral}

Para análise da ocorrência de térmitas, procedeu-se amostragem sistemática em gride com 40 pontos espaçados em 75 m x 75 m, conforme croqui representado na Figura 1.

\section{Figura 1 - Mapa de Amostragem dos pontos-amostras na área de estudo}

Figure 1 - Sampling Map of the sample points in the study area

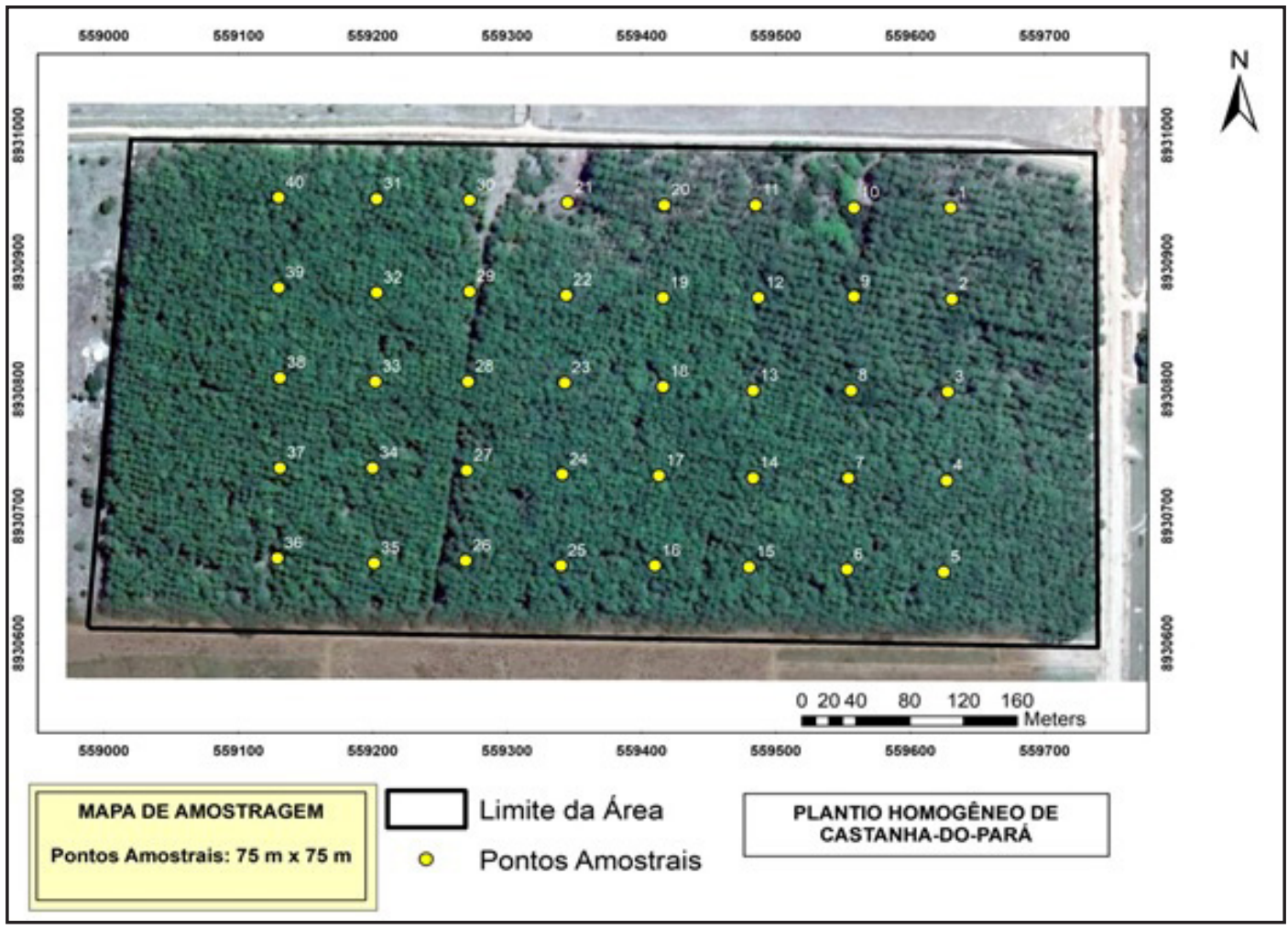

Fonte: Autores (2020)

Em cada ponto amostral utilizou-se uma isca, sem qualquer produto fitossanitário, confeccionada com rolinho de papelão corrugado de $20 \mathrm{~cm}$ de comprimento e $5 \mathrm{~cm}$ de diâmetro, inserido no solo com auxílio de cavadeira, totalizando 40 iscas distribuídas na área de estudo. Devido, sobretudo, às dificuldades e às condições de logística e de acesso à área do plantio, não foi possível a realização de mais de uma coleta durante o ano. Assim, as iscas permaneceram em campo por um período de 40 dias (13/04/2019 a 18/05/2019), quando, então, foram coletadas e acondicionadas em sacos plásticos vedados, devidamente identificados com número sequencial e respectiva coordenada geográfica do ponto de coleta.

Após a coleta do material em campo, as iscas foram avaliadas no Laboratório da Madeira da Universidade do Estado de Mato Grosso; foi também realizada a triagem e a contagem do total de térmitas por isca. Exemplares dos espécimes coletados foram enviados ao Laboratório de Termitologia do Departamento de Zoologia da UnB, em nome do Prof. Dr. Reginaldo Constantino, para identificação em nível de gênero e espécie.

A fim de avaliar a dispersão dos térmitas ocorrentes na área, adotou-se o Índice de Morisita, desenvolvido por Morisita (1959). Para o cálculo, considerou-se como o número de indivíduos a soma dos dois gêneros identificados, Heterotermes e Nasutitermes, obtendo-se um valor de dispersão único, de forma a se identificar possível padrão uniforme, agregado ou 
aleatório. Valores desse índice menor que um indicam um padrão uniforme; valores iguais a um, padrão aleatório; e valores acima de um, padrão agregado.

Complementarmente à análise de distribuição espacial, utilizou-se de representação temática e quantitativa do número de indivíduos por pontos amostrais na área total, a partir da técnica de interpolação spline do software ArcGis 10.1, já que a área amostrada é baseada em pontos equidistantes em relação ao espaço de análise. Além disso, esse algoritmo permite enfatizar os valores no entorno dos pontos amostrais, o que é apropriado para se verificar diferenças mais sensíveis na amostragem.

\section{Resultados e discussão}

Do total de 40 amostras instaladas em campo, em dez iscas verificou-se a ocorrência de térmitas dos gêneros Nasutitermes (DUDLEY, 1890) e Heterotermes (FROGGATT, 1897) (Tabela 1).

\section{Tabela 1 - Análise quantitativa da ocorrência de térmitas em sistema homogêneo de castanha-do-pará no sul da Amazônia}

Table 1 - Quantitative analysis of the occurrence of termites in a homogeneous system of Brazil nut in south Amazon

\begin{tabular}{lccc}
\hline $\mathbf{A m o s t r a}$ & Gênero/espécie & No Indivíduos & Frequência (\%) \\
\hline $\mathbf{0 4}$ & Nasutitermes sp. & 85 & 6,0 \\
$\mathbf{1 0}$ & Heterotermes tenuis & 540 & 38,4 \\
$\mathbf{1 1}$ & Heterotermes tenuis & 390 & 27,8 \\
$\mathbf{1 3}$ & Nasutitermes sp. & 50 & 3,6 \\
$\mathbf{1 4}$ & Nasutitermes sp. & 25 & 1,8 \\
$\mathbf{1 7}$ & Heterotermes tenuis & 1 & 0,1 \\
$\mathbf{1 8}$ & Nasutitermes sp. & 35 & 2,5 \\
$\mathbf{1 9}$ & Nasutitermes sp. & 180 & 12,8 \\
$\mathbf{2 2}$ & Heterotermes tenuis & 78 & 5,6 \\
$\mathbf{2 3}$ & Nasutitermes sp. & 21 & 1,5 \\
\hline Total & & 1405 & $100 \%$ \\
\hline
\end{tabular}

Fonte: Autores (2020)

Ao todo, foram amostrados 1.405 indivíduos, sendo $396(28,1 \%)$ pertencentes ao gênero Nasutitermes e 1.009 (71,8\%) à espécie Heterotermes tenuis (HAGEN, 1858). A pesar da predominância dos indivíduos do gênero Heterotermes, os térmitas do gênero Nasutitermes exploraram um maior número de amostras-iscas (seis) em relação à espécie Heterotermes tenuis (quatro). Em razão do excesso de umidade nas amostras-iscas quando da coleta, houve prejuízo da integridade dos exemplares enviados para identificação, o que limitou a identificação apenas em nível de gênero para Nasutitermes. Observa-se que as amostras de número dez, 11 e 19 apresentaram valores mais expressivos, superiores a 100 indivíduos; essas amostras estão localizadas na borda norte da área e representaram $79 \%$ da amostragem. Importante salientar que, de maneira geral, não há diferenciação entre a cobertura vegetal externa presente nas proximidades das quatro bordas da área, sendo verificadas pastagens em todo o entorno do plantio. 
A baixa riqueza de térmitas no plantio homogêneo de Castanha-do-Pará pode ter sido influenciada principalmente pela forma de amostragem, já que, mesmo avaliando-se um número amplo de pontos amostrais, utilizou-se apenas um único método amostral, a partir do uso de iscas celulósicas para a avaliação geral da área. Além disso, deve-se considerar o fato de parte do material coletado estar compremetido para uma identificação em nível de espécie, já que a coleta foi realizada durante o período chuvoso na região, e o excesso de umidade prejudicou a integridade das amostras para uma identificação mais detalhada das espécies. Dessa forma, os resultados do presente estudo diferem daqueles obtidos por Silva et al. (2015), que avaliaram a ocorrência de térmitas em plantio de eucalipto em Mato Grosso do Sul e obtiveram uma riqueza de 18 espécies e 11 gêneros. Sales et al. (2010), avaliando a frequência e a riqueza de espécies de térmitas que ocorrem em plantações de eucalipto, na região do litoral norte da Bahia, identificaram 21 espécies e 26 gêneros.

Verifica-se que houve domínio de forrageamento entre os gêneros de térmitas, visto que, nos pontos amostrais identificados, não ocorreram indivíduos de Nasutitermes e Heterotermes na mesma amostra-isca. A ocorrência do gênero Nasutitermes nas iscas celulósicas pode ser explicada pelo fato de que esses térmitas apresentam hábitos alimentares variados, podendo ser xilófagos ou ceifadores, mas, principalmente, se alimentam a partir da madeira e serapilheira, além de construírem ninhos epígeos, subterrâneos ou arborícolas (CONSTANTINO, 2015).

A espécie Heterotermes tenuis é reconhecida como sendo de grande importância econômica em sistemas florestais e agrícolas, e sua ocorrência em sistemas de Castanha-do-Pará indica um potencial de danos para essa cultura e ressalta a importância do monitoramento das áreas de produção e de avaliação de possíveis danos nas árvores adultas ou nas fases iniciais de desenvolvimento das plantas. De acordo com Wood (1978), o gênero Heterotermes se alimenta de madeira viva. Essa espécie também possui grande importância em culturas agrícolas, especialmente na cultura da cana-de-açúcar. Em sistemas florestais, Castro et al. (2012), utilizando iscas celulósicas, identificaram a ocorrência de Heterotermes tenuis em plantios de eucalipto no sul de Mato Grosso. Peres Filho et al. (2010), estudando a termitofauna em reflorestamento de eucalipto e mata ripária em Mato Grosso, observaram que a espécie Heterotermes tenuis representou $49 \%$ do total de indivíduos amostrados na área de mata e $79 \%$ no plantio homogêneo. Corassa et al. (2014) avaliaram térmitas associados à degradação de cinco espécies florestais em campo de apodrecimento, no município de Sinop - MT, e verificaram que, entre os térmitas que se alimentam de madeira, o de maior ocorrência foi Heterotermes tenuis, sendo registrado em quatro espécies (Inga sp., Azadirachta indica, Trattinnickia rhoifolia e Bagassa guianensis).

O padrão de distribuição espacial dos térmitas é enquadrado como agregado, já que o valor obtido para o índice de Morisita foi de 9,9. A interpolação das coordenadas geográficas e o número de térmitas nos pontos amostrais, representados na Figura 2, confirmam esse padrão.

A representação temática a partir das medidas de interpolação indica uma maior concentração do número de indivíduos na borda norte da área, onde ocorreram dois pontos amostrais com os maiores valores de abundância de térmitas, representados pelas cores vermelha e alaranjada. Além disso, fica evidente a predominância de pontos amostrais em que não houve ocorrência de térmitas amostrados nas iscas.

A distribuição agregada é o padrão mais comum para térmitas, e essa agregação dos indivíduos ocorreu na área do plantio homogêneo de Castanha-do-Pará, sobretudo na borda norte da área. Ainda que na borda ocorram influências de variáveis ambientais de forma diferente em relação ao interior da área- como maior luminosidade, ventos, umidade e temperatura- é importante mencionar que, nas áreas de maiores valores de abundância (laranja e vermelho), verificou-se a presença de mourões e restos de madeiras dentro da área do plantio, o que possivelmente pode favorecer a disponibilidade de alimento para ambos os gêneros identificados. Nesse sentido, Florencio e Diehl (2006) afirmam que o clima e a disponibilidade local de recursos para alimentação e nidificação se relacionam com a distribuição e a abundância dos organismos. Além disso, as relações intra e interespecíficas, bem como os distúrbios ambientais também 
exercem influência sobre essa dinâmica.

Outros estudos de sistemas florestais homogêneos e pastagens apresentaram resultados similares quanto à distribuição espacial. Peres Filho et al. (2012) avaliaram a distribuição espacial de cupinzeiros em um plantio de Teca (Tectona grandis) e também verificaram dispersão agregada, assim como Dias et al. (2012), ao analisarem a distribuição es pacial de térmitas em área de pastagens.

Figura 2 - Mapa de Amostragem dos pontos-amostras na área de estudo

Figure 2 - Sampling Map of the sample points in the study area

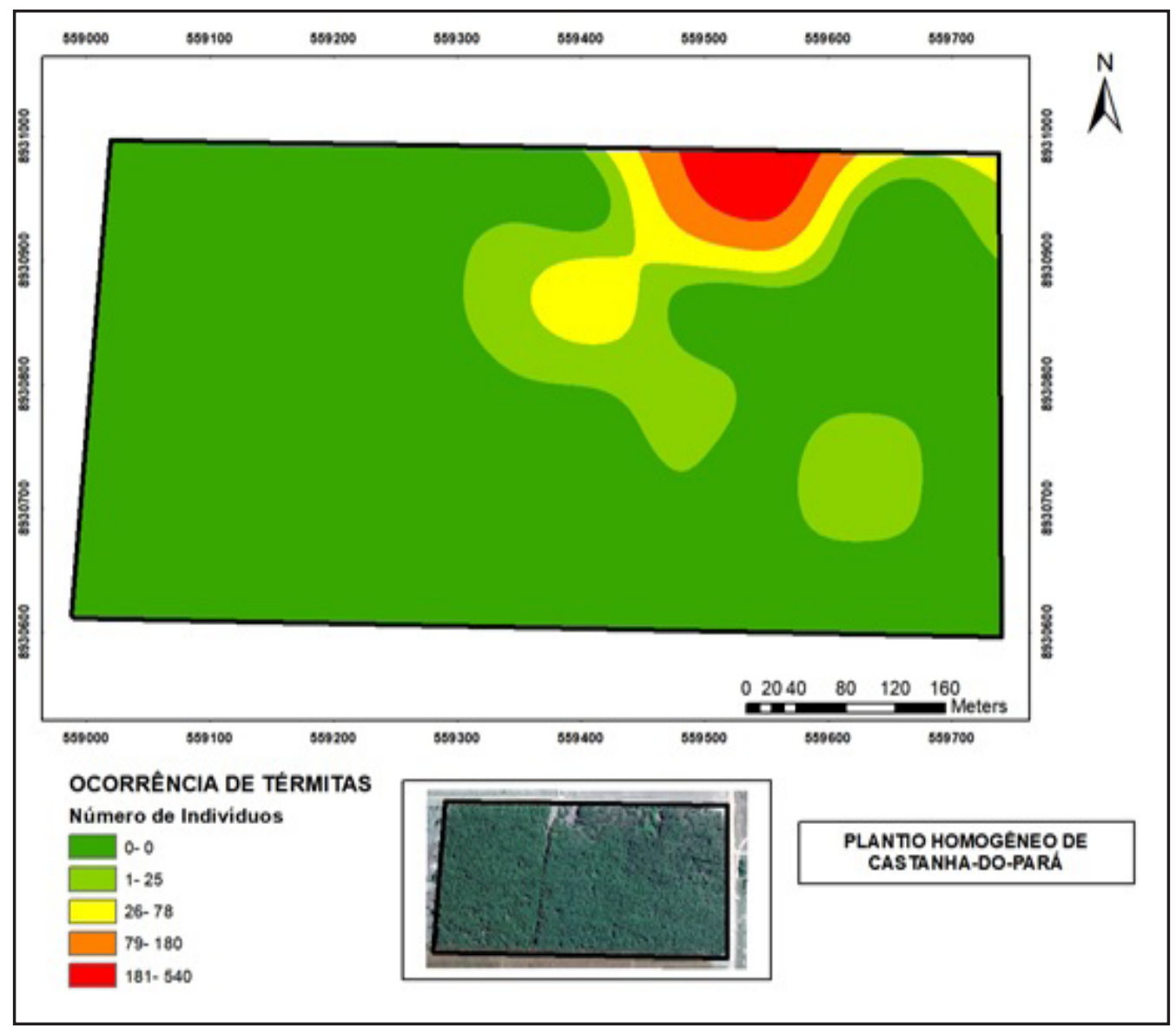

Fonte: Autores (2020)

\section{Conclusões}

A distribuição espacial das térmitas do gênero Nasutitermes e da espécie Heterotermes tenuis, no plantio homogêneo de Castanha-do-Pará, apresenta um padrão agregado.

A ocorrência de Heterotermes tenuis em sistemas de plantio homogêneo de Castanha-doPará deve ser avaliada com importância, já que essa espécie pode se tornar potencial praga na região. Além disso, torna-se fundamental avançar em novos estudos para avaliar possíveis danos dessa espécie em sistemas de produção de Castanha-do-Pará. 


\section{Referências}

ALVARES, C. A.; STAP, J.L.; SENTELHAS, P.C.; GONÇALVES, J.L. de M.; SPAROVEK, G. Koopen's climate classification map for Brazil. Meteorologische Zeitschrift, Stuttgart, v. 22, n. 6, p. 711-728, 2014.

CASTRO, C. K. C.; DORVAL, A.; PERES FILHO, O.; SILVA, A. L.; SOUZA, E. C.; MARQUES, E. N. Forrageamento de Heterotermes tenuis (Hagen) em diferentes profundidades no solo em povoamentos de Eucalyptus spp. no município de Cuiabá-MT. BRAZILIAN JOURNAL OF AGRICULTURE - Revista de Agricultura, Piracicaba, v. 87, n.2, p. 122, 2012.

CONSTANTINO, Reginaldo. Cupins do Cerrado. 1. ed. Rio de Janeiro: Technical Books, 2015.167 p.

CORASSA, J. de N.; PIRES, E.M.; ANDRADE NETO, V. R. de;TARIGA, T. C. Térmitas associados à degradação de cinco espécies florestais em campo de apodrecimento. Floresta e Ambiente, Seropédica, v.21, n.1, p.78-84, 2014.

DIAS, N. P.; MEDEIROS, L. R.; PAZINI, J. de B.; SILVA, F. F. da. Distribuição espacial de Procornitermes sp. (Isoptera: Termitidae) em função das propriedades físicas do solo em área de pastagem no município de São Borja, Rio Grande do Sul. Revista Brasileira de Agroecologia, [s. l.], v.7, n.2, p.104-111, 2012.

FERNANDES, S. E. A castanha do Brasil e oportunidades em Mato Grosso. Disponível em: http://www. mt.gov.br/rss/-/asset_publisher/Hf4xlehM0Iwr/content/id/9433447. Publicado em 15 de março de 2018. Acesso em: 16 set. 2019.

FLORENCIO, D. F.; DIEHL, E. Termitofauna (Insecta, Isoptera) em remanescentes de floresta estacional semidecidual em São Leopoldo, Rio Grande do Sul, Brasil. Revista Brasileira de Entomologia, São Paulo, v.50, n.4, p.505-511, 2006.

HOMMA, A. K. O; MENEZES, A. J. E. A.; MAUÉS, M. M. Castanheira-do-pará: os desafios do extrativismo para plantios agrícolas. Boletim do Museu Paraense Emílio Goeldi. Ciências Naturais, Belém v.9, n.2, p.293306, 2014.

IBGE - INSTITUTO BRASILEIRO DE GEOGRAFIA E ESTATÍSTICA. Produção da Extração Vegetal e da Silvicultura - PEVS 2018. Disponível em: https://www.ibge.gov.br/estatisticas/economicas/agricultura-epecuaria/9105-producao-da-extracao-vegetal-e-da-silvicultura.html?=\&t=resultados. Acesso em: 26 set. 2019.

MORISITA, M. Measuring of the dispersion of individuals and analysis of the distributions patterns. Memoirs of the Faculty of Science, Fukuoka, Japan, v. 2, n.4, p. 215-235, 1959.

PERES FILHO, O.; DORVAL, A.; JANUÁRIO, A. B. S.; ROCHA, J. R. M. Levantamento da termitofauna em reflorestamentos de Eucalyptus camaldulensis e mata ripária no município de Cuiabá, estado de Mato Grosso. Revista Multitemas, Campo Grande, n. 38, p. 7-26, 2010.

PERES FILHO, O.; SOUZA, J.C. de.; SOUZA, M.D. de.; DORVAL, A. Distribuição espacial de cupinzeiros de Cornitermes snyderi (Isoptera: Termitidae) e sua associação com teca. Pesquisa Florestal Brasileira, Colombo, v. 32, n. 70, p. 175-181, 2012.

SALES, M. J. D.; MATOS, W. C.; REIS, Y. T.; RIBEIRO, G. T. Frequência e riqueza de cupins em áreas de plantio de eucalipto no litoral norte da Bahia. Pesquisa Agropecuária Brasileira, Brasília, v.45, n.12, p.13511356, 2010.

SCOLES, R.; CANTO, M. S.; ALMEIDA, R. G.; VIEIRA, D. P. Sobrevivência e frutificação de Bertholletia excelsa Bonpl. em áreas desmatadas em Oriximiná, Pará. Floresta e Ambiente, Seropédica, v. 23, n.4, p. 555-564, 2016.

SILVA, A. P. T. da; CUNHA, H. F. da.; RICARDO, J. A. de D.; ABOT, A. R. Espécies de cupins (Isoptera) em cultura de eucalipto sob diferentes sistemas de manejo de irrigação, em região de transição cerrado-pantanal de Mato Grosso do Sul, Brasil. Revista Árvore, Viçosa, v. 39, p. 137-146, 2015.

WOOD, T. G. Food and feeding habits of termites. In: Production ecology of ants and termites (M.V. Brian, ed.). Cambridge University Press, Cambridge, p.55-80, 1978. 